\title{
ELECTROCHEMICAL ANALYSIS AND THE VOLTAIC SERIES
}

BY J. E. ROOT

When two platinum electrodes are dipped into an electrolytic solution and a difference of potential is created by means of an external electromotive force, only a small current flows when the potential difference is small. This current is known as the residual current, and its magnitude depends on the amount of air dissolved in the electrolyte, on the size of the electrodes, and on the distance between them. While the theory of the residual current is still a matter of discussion, ${ }^{I}$ the current itself is of no importance in regard to analytical determinations, because no measurable change in the electrolyte is produced by it. As the potential difference between the electrodes is increased, the residual current increases, though not rapidly. Above a certain value, which is not clearly marked, the current increases much more rapidly with increasing potential difference than before and the electrolyte undergoes measurable decompo. sition. $^{2}$ The counter-electromotive force of polarization at the moment when this change occurs is known as the decomposition voltage of the solution. The difference of potential as measured on a voltmeter is the decomposition voltage plus the fall of potential in the solution, and therefore depends on the size and distance apart of the electrodes. The decomposition voltage depends on the nature, concentration, and temperature of the solution. The decomposition voltage increases with increasing dilution, but this change is not very large and would rarely exceed $\mathrm{o} . \mathrm{r}$ volt for complete precipitation in most of the solutions used in electrochemical analysis. In general, the decomposition voltages for different solutions vary with the nature of the acid radical and of the basic radical, though the metals which are

1 Bigelow. Jour. Phys. Chem. 7, 327 (1903).

${ }^{2}$ Le Blanc. Zeit. phys. Chem. 8, 299 (I89I). 
instable in presence of water and certain acids form apparent exceptions. It is obviously possible to arrange the salts of the different metals with the same acid radical in the order of decreasing decomposition voltages, each successive metal precipitating at a lower voltage than the one immediately preceding it in the series. Such an arrangement is known as an electrochemical or a voltaic series. It is found experimentally that the order of the metals is not independent of the acid radical. Thus tin stands above copper in the electrochemical series when chlorides are in question and below it in cyanide solutions.

This difference of decomposition voltage is what makes the electrolytic separation of two metals possible. If two metals precipitate from a given solution as two phases, each phase consisting of one of the metals pure, then we can separate these two metals electrolytically by electrolyzing under a difference of potential sufficient to precipitate one metal and not to precipitate the other. This is feasible practically only when there is a difference of at least $0.2-0.3$ volt between the decomposition voltages of the two salts. When there is only a very slight difference between the decomposition voltages, no separation can be made. When the difference of decomposition voltage is at least 0.3 volt, a separation is possible unless the metals can precipitate as a single phase. This is a contingency which must always be kept in mind. In concentrated cyanide solutions, the decomposition voltage for silver is nearly $\mathrm{I}$ volt higher than that for mercury, but some silver precipitates as amalgam when a mixture of the mercury and silver salts is subjected to electrolysis.

When the decomposition voltages are markedly different and when the two metals cannot separate as a single phase, a separation is always possible; but there are two ways of carrying this out which may be called the constant voltage and the constant current methods. In constant voltage separations, the voltage is kept constant at a value just below that at which the second metal can precipitate. The initial current is then relatively high and decreases as the first metal precipitates, 
reaching the value of the residual current when the first metal has entirely precipitated. This method has several distinct advantages. It is of universal application. Since the second metal cannot precipitate, by hypothesis, the current can be allowed to run for an indefinite period if desired. From the magnitude of the current, some information can be obtained as to the amount of metal which is yet to be precipitated. The constant voltage separation was first introduced as a general method by Freudenberg. It is not, however, in general use. The theory of electrochemical analysis is of comparatively recent development, and the practice proceeded along lines which were simpler experimentally though not theoretically.

Ever since the time of Faraday the chemist has been familiar with the voltameter. The measurement of current by means of the voltameter is a chemical measurement and the current is the only one of the three electrical units, volt, ampere, ohm, which can be measured readily with the appliances to be found in every chemical laboratory. Since the current measurement was the only one that the chemist could make easily, it was natural for him to hold the current constant, or approximately constant, and thus to develop the constant current methods of separation. We shall therefore find that practically all the standard methods of separation are constant current methods and we must consider what conditions are essential for a separation with constant current. The initial current must be higher than the residual current, otherwise there would be no precipitation at all. Since it would not be practical to break the circuit at the very moment when the first metal was completely precipitated, some device must be found which will per-" mit the passage of a current higher than the residual current, which shall prevent the second metal from precipitating, and which shall not alter the weight of the deposit. At first it seems impossible to satisfy such requirements; but this is easily done if the ion that is discharged is either volatile or soluble in the solution. If the decomposition voltage of hydrogen lies be-

${ }^{1}$ Zeit. phys. Chem. I2, 97 ( 1893 ). 
tween that of the two metals, hydrogen will be set free instead of the second metal. This is the usual way in which the difficulty is solved, but it is not the only way in which it might be overcome. Suppose we wish to separate copper from iron, both metals being present as sulphates. We can either make the solution so acid that the iron cannot precipitate under the conditions of the experiment, or we can change the iron into the ferric state and no precipitation of iron will occur until the current has changed the ferric salt back into ferrous salt. This latter method, while theoretically sound, is less generally applicable than the other and need not be considered further. The essential condition, then, for a constant current separation is that the decomposition voltage for hydrogen shall lie below that of the second metal. While one object to be obtained in selecting one solution for a given separation rather than another is to have the decomposition voltages for the two metals as different as possible, the second very important object is to obtain a solution in which the decomposition voltage for hydrogen lies below that of one of the metals. In any given solution, the metals, whose decomposition voltages lie below that of hydrogen, can be separated from each other only by a constant voltage method, while any metal of this group can be separated from any of the other metals by a constant current method except in so far as the possible precipitation of the two metals as one phase may affect the situation.

This distinction between constant voltage and constant current separations was seen clearly by Freudenburg, ${ }^{\text {T }}$ who first discusses the analogy between the precipitation of metals by the current and by hydrogen sulphide and then goes on:

"The behavior of the metals with reference to the potentials of hydrogen in different solutions is what has made the simplest separations possible and the earlier methods depend on it almost exclusively. Since it is not necessary in those cases to maintain a definite potential difference, it was very easy for the importance of this to be completely overlooked. Up to now

${ }^{1}$ Zeit. phys. Chem, 12, 103 ( 1893 ). 
people have used the same potential difference for all precipitations and, by means of resistances, have varied the current density in such a way as to keep the evolution of gas from affecting the precipitate." This same distinction is recognized less clearly by Neumann: "The separation of certain metals from others may be effected by the addition of strong mineral acids to their salt solutions. In this way the deposition of iron, cobalt, nickel; cadmium, and zinc is prevented. Since those metals are in all cases first separated for which the least electromotive force is required, it will be the noble metals, gold, silver, copper, and mercury that are first deposited; while if a considerable excess of acid be present, the remainder of the series of metals given on p. 35 will not be deposited, a liberation of the hydrogen of the acid being produced instead." The significance of the constant current separation is here recognized only for the case of acid solutions, and there is no clear appreciation of the importance of a general classification based on the intermediate evolution of hydrogen. The other text-books on electrochemical analysis are still more vague in regard to the principles on which the separations depend. At the time I began this work there were no data which would enable one to make a proper classification for many of the solutions ordinarily used in electrochemical separations.

While a determination of the decomposition voltages in different solutions would in itself have given the necessary data for such a classification, it would have given nothing more. Instead of making a series of such determinations, it was therefore thought best to measure the potential differences and currents under the conditions of actual analytical work, with the platinum electrodes actually employed. While the potential differences at which the metals precipitate differ from the true decomposition voltages by the fall of potential through the liquid, these values are the limiting values which must not be exceeded in a constant voltage separation. In this way data are obtained as to the order of magnitude of the residual current.

1 Electrolytic Methods of Analysis, p. 166. 
In the following experiments, the same anode and the same cathode were always used, and they were always the same distance apart. The concentrations and temperatures are given in each case so that the conditions are all clearly defined and can easily be reproduced. The cathode was a Classen platinum dish $9.4 \mathrm{~cm}$ in diameter and $4.7 \mathrm{~cm}$ deep. The wetted surface was approximately $145 \mathrm{~cm}^{2}$ when there were $200 \mathrm{cc}$ of solution in the dish and I2 I $\mathrm{cm}^{2}$ when there were $150 \mathrm{cc}$. The anode was a perforated Classen disc $4.5 \mathrm{~cm}$ in diameter. The distance between the anode and cathode was I $\mathrm{cm}$ and was measured as follows. The anode was placed on the bottom of the dish and then raised vertically $\mathrm{I} \mathrm{cm}$ and clamped in position. A mark on the stem of the electrode made it possible to adjust the electrode anew. A sheet of asbestos board on a tripod was placed beneath the dish and so arranged as to permit of the easy heating of the electrolyte by means of a Bunsen burner. All measurements were made either at $20^{\circ}$ or at $60^{\circ}$. Since the value of the residual current is a function of the time, all readings were made three minutes after closing the circuit. The current was obtained from storage cells and the difference of potential between the anode and the cathode was regulated by means of a variable resistance placed in series. The current was measured with a Weston milli-ammeter and the potential difference with a Weston voltmeter, the current readings being taken only while the voltmeter circuit was open. The chemicals for the experiments were taken from the laboratory stock and were not purified further.

The following stock solutions were prepared, each containing $0.2 \mathrm{~g}$ metal per $50 \mathrm{cc}$ : copper sulphate, lead nitrate, nickel sulphate, cadmium sulphate, zinc sulphate, mercuric chloride, silver nitrate. The bismuth nitrate solution contained about $0.2 \mathrm{~g} \mathrm{Bi}$ per $\mathrm{I} 2 \mathrm{cc}$ and enough free nitric acid to prevent precipitation. The antimony trichloride solution was approximately $25 \mathrm{~g} \mathrm{SbCl}_{3}$ and $250 \mathrm{cc}$ dilute $\mathrm{HCl}$ per liter, or about $0.2 \mathrm{~g} \mathrm{Sb}$ per I $6 \mathrm{cc}$. The ammonium oxalate solution was saturated at $20^{\circ}$. The $\mathrm{Na}_{2} \mathrm{HPO}_{4}$ solution contained $90 \mathrm{~g}$ crystallized sodium phosphate per liter and had a specific gravity of 1.025 at $20^{\circ}$. The 
phosphoric acid solution was made by diluting I25 g glacial acid to one liter and its specific gravity was I.3. The ammonia solution had a specific gravity of 0.92 at $20^{\circ}$ and the sodium hydroxide solution one of I.28. The potassium cyanide solution contained $260.4 \mathrm{~g} \mathrm{KCN}$ per liter $(4 N)$ and was kept in a tightly-stoppered bottle away from the air.

In Tables I-XXXIX are given the experiments with cyanide, oxalate, and phosphate solutions. Lead cyanide is insoluble in hot and cold cyanide solutions. At $60^{\circ}$ a precipitate occurs in the alkaline citrate cyanide solution of bismuth. Silver oxalate does not dissolve in an excess of ammonium oxalate.

TABLE I.

Solution: $\mathrm{N} / 4 \mathrm{KCN}$

Vol. 200 cc. Temp. $20^{\circ}$

\begin{tabular}{l|c||c|c}
\hline \hline Volts & Milli-amperes & Volts & Milli-amperes \\
\hline 0.2 & - & 2.45 & 22 \\
0.58 & 0.05 & 2.74 & $44^{1}$ \\
$0.8 \mathrm{I}$ & 0.1 & 3.0 & 87 \\
1.0 & $0.2 \mathrm{I}$ & 3.36 & 185 \\
1.32 & $\mathrm{I} .4$ & 3.65 & $35^{2}$ \\
1.5 & 2.3 & 3.9 & 530 \\
1.8 & 4.3 & 4.3 & 850 \\
2.0 & 5.5 & $4.5 \mathrm{I}$ & 1050 \\
\hline
\end{tabular}

${ }^{1}$ Slight bubbles at cathode.

${ }^{2}$ Solution turns yellow round the anode, probably owing to presence of paracyanogen.

TABLE II.

Solution: $\mathrm{N} / 4 \mathrm{KCN}+0.2 \mathrm{~g} \mathrm{Ag}$ as $\mathrm{AgNO}_{3}$

Vol. $200 \mathrm{cc}$. Temperature $20^{\circ}$

\begin{tabular}{c|c||c|c}
\hline \hline Volts & Milli-amperes & Volts & Milli-amperes \\
\hline 0.8 & 0.2 & 2.83 & $70^{2}$ \\
1.05 & 0.4 & 3.14 & 145 \\
1.4 & 1.85 & 3.55 & 385 \\
1.7 & 6.1 & 3.77 & 500 \\
2.04 & $15^{1}$ & 4.0 & 770 \\
2.48 & 25 & 4.28 & IOOO
\end{tabular}

${ }_{1}$ Deposit of silver.

2 Few bubbles at anode. Solution slightly yellow at end of run. 
TABLE III.

Solution: $\mathrm{N} / 4 \mathrm{KCN}+0.2 \mathrm{~g} \mathrm{Hg}$ as $\mathrm{HgCl}_{2}$

Vol. 200 cc. Temp. $20^{\circ}$

\begin{tabular}{c|c|c|c}
\hline \hline Volts & Milli-amperes & Volts & Milli-amperes \\
\hline I.3 & I.3 & 3.6 & I20 \\
I.8 & I8 & 4.0 & 225 \\
2.2 & $30^{1}$ & 4.2 & 350 \\
2.5 & 35 & 4.55 & 600 \\
2.7 & 60 & 4.75 & 800 \\
3.2 & 70 & 5.15 & I I 50 \\
\hline
\end{tabular}

1 Slight deposit of mercury at cathode; few bubbles at anode.

2 Bubbles at both electrodes.

TABLE IV.

Solution : $\mathrm{N} / 4 \mathrm{KCN}+0.2 \mathrm{~g} \mathrm{Bi}$ as $\mathrm{Bi}\left(\mathrm{NO}_{3}\right)_{3}+4 \mathrm{~g}$ cryst. citric acid $+\mathrm{NaOH}$ in slight excess

Vol. 200 cc. Temp. $20^{\circ}$

\begin{tabular}{l|c|c|c}
\hline Volts & Milli-amperes & Volts & Milli-amperes \\
\cline { 1 - 2 } I.22 & I.I & 2.75 & $35^{3}$ \\
I.5 & I.8 & 3.25 & 90 \\
2.0 & $8.5^{1}$ & 3.66 & 410 \\
2.5 & $25^{2}$ & 3.9 & 475 \\
\hline
\end{tabular}

1 Anode turns brown.

${ }^{2}$ Bubbles at anode; cathode turns black.

${ }^{3}$ Heavy deposit of metal at cathode, no bubbles. Metal black and does not adhere well.

TABLE $V$.

Solution: $\mathrm{N} / 4 \mathrm{KCN}+0.2 \mathrm{~g} \mathrm{Cd}$ as $\mathrm{CdSO}_{4}$

Vol. $200 \mathrm{cc}$. Temp. $20^{\circ}$

\begin{tabular}{|c|c|c|c|}
\hline Volts & Milli-amperes & Volts & Milli-amperes \\
\hline I. 6 & 3.0 & 4.0 & $175^{3}$ \\
\hline 2. I & ro & $4 \cdot 5$ & 375 \\
\hline 2.5 & 20 & 4.96 & 650 \\
\hline 2.8 & $55^{1}$ & $5 . \mathrm{I}$ & 750 \\
\hline $3 \cdot 3.5$ & $85^{2}$ & $5 \cdot 3$ & 950 \\
\hline 3.75 & I 20 & 6.0 & I 500 \\
\hline
\end{tabular}

${ }^{1}$ Slight deposit of metal.

${ }^{2}$ Appearance of bubbles at anode.

3 Bubbles at both electrodes. 
TABLE VI.

Solution: $\mathrm{N} / 4 \mathrm{KCN}+0.2 \mathrm{~g} \mathrm{Zn}$ as $\mathrm{ZnSO}_{4}$

Vol. $200 \mathrm{cc}$. Temp. $20^{\circ}$

\begin{tabular}{l|c||c|c}
\hline \hline Volts & Milli-amperes & Volts & Milli-amperes \\
\cline { 2 - 3 } I.25 & I.O & 3.1 & I 35 \\
I.55 & 2.5 & 3.6 & 305 \\
2.0 & 6.5 & 3.85 & 475 \\
2.4 & 20 & 4.0 & 570 \\
2.85 & 70 & 4.5 & 900
\end{tabular}

${ }^{1}$ No zinc was deposited. In this run, as in some of the others, no record was kept of the voltage at which hydrogen appeared.

TABLE VII.

Solution : $\mathrm{N} / 4 \mathrm{KCN}+0.2 \mathrm{~g} \mathrm{Ni}$ as $\mathrm{NiSO}_{4}$

Vol. $200 \mathrm{cc}$. Temp. $20^{\circ}$.

\begin{tabular}{l|c||c|c}
\hline Volts & Milli-amperes & Volts & Milli-amperes \\
\cline { 1 - 2 } 1.0 & $2 . \mathrm{I}$ & 2.8 & 350 \\
I.58 & IO & $3 . \mathrm{I}$ & 455 \\
1.69 & II & 3.43 & 650 \\
2.08 & 80 & 3.8 & 820 \\
2.35 & I $70^{1}$ & 4.2 & $1050^{2}$
\end{tabular}

1 Traces of bubbles al cathode.

2No nickel deposited.

TABLE VIII.

Solution: $\mathrm{N} / 4 \mathrm{KCN}+0.2 \mathrm{~g} \mathrm{Cu}$ as $\mathrm{CuSO}_{4}$ Vol. $200 \mathrm{cc}$. Temp. $20^{\circ}$

\begin{tabular}{l|c||c|c}
\hline \hline Volts & Milli-amperes & Volts & Milli-amperes \\
\hline 0.74 & 0.5 & 2.28 & 390 \\
1.0 & 2.4 & 2.5 & 520 \\
1.3 & 10 & 2.85 & 680 \\
1.6 & 60 & 3.35 & 770 \\
1.75 & $145^{1}$ & 4.0 & 850 \\
2.0 & 265 & 4.4 & $1000^{2}$
\end{tabular}

${ }^{1}$ Bubbles appear.

${ }^{2}$ No copper deposited. 
TABLE IX.

Solution: $\mathrm{N} / 4 \mathrm{KCN}$

Vol. $200 \mathrm{cc}$. Temp. $60^{\circ}$

\begin{tabular}{l|l||l|r}
\hline \hline Volts & Milli-amperes & Volts & Milli-amperes \\
\hline 0.2 & 0.0 & 2.5 & $75^{1}$ \\
0.48 & 0.1 & 2.82 & 165 \\
0.66 & 0.2 & 3.12 & 293 \\
1.0 & 1.25 & 3.4 & 560 \\
1.23 & 4.0 & 3.67 & 760 \\
1.7 & 10 & 3.9 & 1010 \\
1.94 & 15 & 4.15 & 1280 \\
2.2 & 28 & &
\end{tabular}

${ }^{1}$ Slight bubbles at cathode.

TABLE X.

Solution: $\mathrm{N} / 4 \mathrm{KCN}+0.2 \mathrm{~g} \mathrm{Ag}$ as $\mathrm{AgNO}_{3}$ Vol. 200 cc. Temp. $60^{\circ}$

\begin{tabular}{l|c||c|c}
\hline \hline Volts & Milli-amperes & Volts & Milli-amperes \\
\cline { 2 - 3 } I.I & I.4 & 2.7 & I $35^{3}$ \\
I.4 & 5.5 & 3.05 & 240 \\
I.8 & I $7^{1}$ & 3.34 & 465 \\
2.14 & $65^{2}$ & 3.5 & 620 \\
2.4 & I I5 & 3.8 & IO20
\end{tabular}

${ }^{1}$ Slight deposit of silver.

2 Heavy deposit of silver.

${ }^{8}$ Bubbles at anode.

TABLE XI.

Solution : $\mathrm{N} / 4 \mathrm{KCN}+0.2 \mathrm{~g} \mathrm{Hg}$ as $\mathrm{HgCl}_{2}$ Vol. $200 \mathrm{cc}$. Temp. $60^{\circ}$

\begin{tabular}{l|c||c|c}
\hline \hline Volts & Milli-amperes & Volts & Milli-amperes \\
\cline { 3 - 4 } O.76 & I.O & 2.85 & I 25 \\
I.I & I.6 & 3.05 & 140 \\
I.46 & 6.3 & 3.4 & 240 \\
I.75 & I5 & 3.7 & 380 \\
2.0 & $60^{1}$ & 4.0 & 650 \\
2.35 & IIO & 4.4 & IOOO
\end{tabular}

${ }^{1}$ Slight deposit of metal.

${ }^{2}$ Bubbles seen at anode. 
TABLE XII.

Solution: $\mathrm{N} / 4 \mathrm{KCN}+0.2 \mathrm{~g} \mathrm{Cd}$ as $\mathrm{CdSO}_{4}$

Vol. $200 \mathrm{cc}$. Temp. $60^{\circ}$

\begin{tabular}{|c|c|c|c|}
\hline Volts & Milli-amperes & Volts & Milli-amperes \\
\hline I. I 5 & 2.5 & 3.15 & 185 \\
\hline I. 55 & $7 \cdot 5$ & 3.7 & 200 \\
\hline 1.8 & IO & 3.9 & 250 \\
\hline $2 . I$ & I 3 & 4.2 & 375 \\
\hline 2.5 & 55 & 4.5 & 680 \\
\hline 2.82 & $125^{1}$ & 4.65 & 850 \\
\hline
\end{tabular}

${ }^{1}$ Deposit of metal; bubbles at anode.

TABLE XIII.

Solution: $\mathrm{N} / 4 \mathrm{KCN}+0.2 \mathrm{~g} \mathrm{Ni}$ as $\mathrm{NiSO}_{4}$

Vol. 200 cc. Temp. $60^{\circ}$

\begin{tabular}{l|c||c|c}
\hline \hline Volts & Milli-amperes & Volts & Milli-amperes \\
\cline { 2 - 3 } & 2.8 & 2.2 & 190 \\
O.9 & 4.3 & 2.57 & 468 \\
I. 5 & 7.3 & 2.8 & 870 \\
I.5 & $90^{1}$ & 3.5 & 1250 \\
2.0 & &
\end{tabular}

1 Bubbles.

No nickel is deposited.

TABLE XIV.

Solution: $\mathrm{N} / 4 \mathrm{KCN}+0.2 \mathrm{~g} \mathrm{Zn}$ as $\mathrm{ZnSO}_{4}$

Vol. 200 cc. Temp. $60^{\circ}$

\begin{tabular}{l|c||l|c}
\hline Volts & Milli-amperes & Volts & Milli-amperes \\
\cline { 2 - 3 } I.25 & 4.1 & 2.8 & $90^{2}$ \\
I.7 & 7.8 & 3.27 & 250 \\
2.0 & 12 & 3.95 & 500 \\
2.39 & $25^{1}$ & 4.1 & I I00
\end{tabular}

${ }^{1} \mathrm{~A}$ few bubbles at anode.

${ }^{2}$ More bubbles.

No zinc is deposited. 
TABLE XV.

Solution : $\mathrm{N} / 4 \mathrm{KCN}+0.2 \mathrm{~g} \mathrm{Cu}$ as $\mathrm{CuSO}_{4}$

Vol. $200 \mathrm{cc}$. Temp. $60^{\circ}$

\begin{tabular}{c|c||c|c}
\hline \hline Volts & Mifli-amperes & Volts & Milli-amperes \\
\hline & I.55 & 2.2 & 550 \\
0.74 & 3.1 & 2.6 & $840^{3 .}$ \\
0.93 & 23 & 3.0 & $1050^{4}$ \\
1.26 & $110^{1}$ & 3.27 & I 300 \\
1.55 & $300^{2}$ & 3.5 & I600 \\
1.9 & &
\end{tabular}

1 Few bubbles at anode.

${ }^{2}$ Bubbles at both electrodes.

${ }^{3}$ Slight deposit of copper, which rapidly dissolves if current is broken.

${ }^{4}$ Heavy deposit of copper dissolving when circuit is broken, with marked counter-electromotive force.

\section{TABLE XVI.}

Solution : I $50 \mathrm{cc}$ sat. ammonium oxalate

Vol. 175 cc. Temp. $60^{\circ}$

\begin{tabular}{l|c||c|c}
\hline \hline Volts & Milli-amperes & Volts & Milli-amperes \\
\cline { 2 - 4 } & I & I.25 & 40 \\
0.4 & 6 & 1.45 & $125^{1}$ \\
0.85 & IO & I.8 & 460
\end{tabular}

${ }^{1}$ Bubbles at both electrodes.

TABLE XVII.

Solution : $150 \mathrm{cc}$ sat. ammonium oxalate $+0.1 \mathrm{~g} \mathrm{Hg}$ as $\mathrm{HgCl}_{2}$ Vol. $175 \mathrm{cc}$. Temp. $60^{\circ}$

\begin{tabular}{l|c||l|c}
\hline \hline Volts & Milli-amperes & Volts & Milli-amperes \\
\cline { 2 - 3 } & 2 & I.22 & 48 \\
0.2 & I 5 & 1.5 & 70 \\
0.4 & $40^{1}$ & I.85 & $220^{8}$ \\
0.9 & $45^{2}$ & 2.15 & 500
\end{tabular}

${ }^{1}$ Slight deposit of metal; no bubbles at anode.

2 Bubbles at anode.

${ }^{3}$ Bubbles at cathode.

Mercuric oxalate is also soluble at $20^{\circ}$ in an excess of ammonium oxalate. 
TABLE XVIII.

Solution : I $50 \mathrm{cc}$ sat. ammonium oxalate + o.I $\mathrm{g} \mathrm{Cu}$ as $\mathrm{CuSO}_{4}$ Vol. $175 \mathrm{cc}$. Temp. $60^{\circ}$

\begin{tabular}{l|c|c|c}
\hline Volts & Milli-amperes & Volts & Milli-amperes \\
\hline O.4 & 4 & I.8 & $350^{2}$ \\
I.O & I IO $^{1}$ & 2.15 & 920 \\
1.45 & I50 & 2.9 & I 900
\end{tabular}

${ }^{1}$ Slight deposit of metal ; bubbles at anode.

${ }^{2}$ Some bubbles at cathode.

Copper oxalate is also soluble at $20^{\circ}$.

TABLE XIX.

Solution : I70 cc sat. ammonium oxalate + O.I $\mathrm{g} \mathrm{Bi}$ as $\mathrm{Bi}\left(\mathrm{NO}_{3}\right)_{3}$ Vol. $176 \mathrm{cc}$. Temp. $60^{\circ}$

\begin{tabular}{c|c||c|c}
\hline \hline Volts & Milli-amperes & Volts & Milli-amperes \\
\hline 0.2 & 0.9 & 1.15 & $60^{1}$ \\
0.4 & 3.5 & 1.5 & 160 \\
0.8 & 20 & 1.8 & $370^{2}$
\end{tabular}

${ }^{3}$ Deposit of metal ; bubbles at anode.

${ }^{2}$ Bubbles at cathode.

Bismuth oxalate does not dissolve at $20^{\circ}$.

\section{TABLE XX.}

Solution : $170 \mathrm{cc}$ sat. ammonium oxalate + o. I $\mathrm{g} \mathrm{Sb}$ as $\mathrm{SbCl}_{3}$ Vol. $178 \mathrm{cc}$. Temp. $60^{\circ}$

\begin{tabular}{|c|c|c|c|}
\hline Volts & Milli-amperes & Volts & Milli-amperes \\
\hline 0.2 & 0.9 & I. 2 & $65^{1}$ \\
\hline 0.4 & 7 & 1.7 & 210 \\
\hline 0.8 & I 5 & 2.0 & 400 \\
\hline
\end{tabular}

${ }^{1}$ Deposit of metal; bubbles at anode.

A good deposit is obtained at the highest current.

Antimony oxalate is soluble at $20^{\circ}$. 
TABLE XXI.

Solution: I50 ce sat. ammonium oxalate + I g Pb as $\mathrm{Pb}\left(\mathrm{NO}_{8}\right)_{2}$ Vol. $175 \mathrm{cc}$. Temp. $60^{\circ}$

\begin{tabular}{l|c|c|c}
\hline Volts & Milli-amperes & Volts & Milli-amperes \\
\hline 0.8 & I I & I.8 & I25 \\
I.25 & 30 & 2.0 & I 75 \\
I.55 & $80^{1}$ & 2.3 & $375^{2}$
\end{tabular}

${ }^{1}$ Deposit of metal; bubbles at anode.

2 Bubbles at cathode.

Lead oxalate does not dissolve at $20^{\circ}$.

TABLE XXII.

Solution : $150 \mathrm{cc}$ sat. ammonium oxalate + o. I $\mathrm{g} \mathrm{Cd}$ as $\mathrm{CdSO}_{4}$

Vol. $175 \mathrm{cc}$. Temp. $60^{\circ}$

\begin{tabular}{c|c|c|c}
\hline \hline Volts & Milli-amperes & Volts & Milli-amperes \\
\hline 0.4 & 0.5 & I.8 & I I 5 \\
0.88 & 6.5 & 2.0 & I75 \\
I.27 & I2 & 3.5 & 350
\end{tabular}

1 Deposit of metal; bubbles at anode.

Cadmium oxalate does not dissolve at $20^{\circ}$.

TABLE XXIII.

Solution : $150 \mathrm{cc}$ sat. ammonium oxalate + O.I g Ni as $\mathrm{NiSO}_{4}$ Vol. $175 \mathrm{cc}$. Temp. $60^{\circ}$

\begin{tabular}{c|c|c|c}
\hline Volts & Milli-amperes & Volts & Milli-amperes \\
\hline I.0 & I2 & 1.9 & $400^{3}$ \\
1.3 & 60 & 2.35 & 750 \\
1.4 & $100^{1}$ & 2.75 & 900 \\
1.6 & $200^{2}$ & 3.3 & 1600
\end{tabular}

1 Bubbles at anode.

2 Bubbles at cathode.

${ }^{3}$ Deposit of metal.

Nickel oxalate is soluble at $20^{\circ}$. 


\section{TABLE XXIV.}

Solution : I 50 cc sat. ammonium oxalate + o. I g Co as $\mathrm{CoSO}_{4}$ Vol. I79 cc. Temp. $60^{\circ}$

\begin{tabular}{c|c|c|c}
\hline Volts & Milli-amperes & Volts & Milli-amperes \\
\hline 0.4 & 6.9 & 1.4 & I $58^{1}$ \\
I.O & I5 & I.65 & 250 \\
I.2 & 55 & I.9 & $425^{2}$
\end{tabular}

${ }^{1}$ Bubbles at both electrodes.

${ }^{2}$ Slight deposit of metal which dissolves rapidly on breaking the circuit. Cobalt oxalate is soluble at $20^{\circ}$.

TABLE XXV.

Solution : I $50 \mathrm{cc}$ sat. ammonium oxalate + o. $\mathrm{g} \mathrm{Fe}$ as $\mathrm{FeSO}_{4}$ Vol. I75 cc. Temp. $60^{\circ}$

\begin{tabular}{c|c||c|c}
\hline \hline Volts & Milli-amperes & Volts & Milli-amperes \\
\hline I.25 & 40 & I.6 & 255 \\
I.45 & $\mathrm{I} 35^{1}$ & 1.9 & $450^{2}$
\end{tabular}

1 Bubbles at both electrodes.

${ }^{2}$ Slight deposit of metal ; dissolving rapidly when circuit is broken. Iron oxalate is soluble at $20^{\circ}$.

TABLE XXVI.

Solution : I $50 \mathrm{cc}$ sat. ammonium oxalate +0 . I g Sn as $\mathrm{SnCl}_{2}$ Vol. 175 cc. Temp. $60^{\circ}$

\begin{tabular}{c|c|c|c}
\hline \hline Volts & Milli-amperes & Volts & Milli-amperes \\
\hline 0.4 & I2 & I.6 & 200 \\
0.8 & I8 & 2.0 & 500 \\
I.3 & I $20^{1}$ & 2.1 & $600^{2}$
\end{tabular}

${ }^{1}$ Bubbles at both electrodes.

2 Slight deposit of metal.

Tin oxalate does not dissolve at $20^{\circ}$. 
TABLE XXVII.

Solution : ${ }_{5} 0 \mathrm{cc}$ sat. ammonium oxalate +0 . I g $\mathrm{Zn}$ as $\mathrm{ZnSO}_{4}$ Vol. $175 \mathrm{cc}$. Temp. $60^{\circ}$. Cathode copper-plated

\begin{tabular}{c|c||c|c}
\hline \hline Volts & Milli-amperes & Volts & Milli-amperes \\
\hline 0.2 & Reverse current & I.6 & $75^{1}$ \\
0.4 & 0 & 1.85 & 210 \\
0.8 & 3.5 & 2.1 & 525 \\
I.25 & 18 & 2.6 & $1400^{2}$
\end{tabular}

1 Bubbles at anode.

2 Deposit of metal.

Zinc oxalate is soluble at $20^{\circ}$.

TABLE XXVIII.

Solution: $50 \mathrm{cc} \mathrm{Na} 2 \mathrm{HPO}_{4}+{ }_{15} \mathrm{cc} \mathrm{H}_{3} \mathrm{PO}_{4}$

Vol. 150 cc. Temp. $60^{\circ}$

\begin{tabular}{c|c||c|c}
\hline Volts & Milli-amperes & Volts & Milli-amperes \\
\cline { 2 - 4 } 0.4 & 0.1 & I.5 & I3 \\
0.8 & 0.4 & 1.9 & $75^{1}$ \\
I.2 & 6.5 & 2.2 & 240 \\
I.3 & IO & 2.5 & 475
\end{tabular}

1 Bubbles at both electrodes.

TABLE XXIX.

Solution: $50 \mathrm{cc} \mathrm{Na} \mathrm{HPO}_{4}+\mathrm{I}_{5} \mathrm{cc}_{3} \mathrm{PO}_{4}+$ O.I g Ag as $\mathrm{AgNO}_{3}$ Vol. $150 \mathrm{cc}$. Temp. $60^{\circ}$

\begin{tabular}{c|c||c|c}
\hline Volts & Milli-amperes & Volts & Milli-amperes \\
\hline 0.4 & 0.2 & 1.2 & $45^{1}$ \\
0.8 & 0.4 & 1.9 & 70
\end{tabular}

1 Deposit of metal; bubbles at anode.

Silver phosphate is readily soluble at $20^{\circ}$ in the amount of phosphoric acid added. 
TABLE XXX.

Solution: $50 \mathrm{cc} \mathrm{Na}_{2} \mathrm{HPO}_{4}+{ }_{15} \mathrm{cc}_{3} \mathrm{PO}_{4}+$ O.I g Hg as $\mathrm{HgCl}_{2}$ Vol. 150 cc. Temp. $60^{\circ}$

\begin{tabular}{c|c||c|c}
\hline \hline Volts & Milli-amperes & Volts & Milli-amperes \\
\hline 0.4 & 0.1 & 1.3 & $45^{1}$ \\
0.8 & 0.3 & 1.4 & 60 \\
1.2 & 18 & 1.7 & 65
\end{tabular}

${ }^{1}$ Deposit of metal; bubbles at anode. Current decreases rapidly as metal precipitates. Deposit is black and powdery; not very adherent. The mercury phosphate redissolves in the sodium phosphate before the phosphoric acid is added.

TABLE XXXI.

Solution: $50 \mathrm{cc} \mathrm{Na}_{2} \mathrm{HPO}_{4}+\mathrm{I}_{5} \mathrm{cc}_{3} \mathrm{PO}_{4}+$ O. I g Cu as CuSO, Vol. 150 cc. Temp. $60^{\circ}$

\begin{tabular}{c|c||c|c}
\hline Volts & Milli-amperes & Volts & Milli-amperes \\
\hline 0.4 & 0.15 & I.75 & I I $5^{1}$ \\
0.8 & 0.4 & 2.15 & I65 \\
I.4 & I2 & 2.55 & 500
\end{tabular}

${ }^{1}$ Deposit of metal; bubbles at anode.

TABIE XXXII.

Solution : $50 \mathrm{cc} \mathrm{Na} \mathrm{HPO}_{4}+20 \mathrm{cc} \mathrm{H}_{8} \mathrm{PO}_{4}+0.1 \mathrm{~g} \mathrm{Bi}$ as $\mathrm{Bi}\left(\mathrm{NO}_{8}\right)_{8}$ Vol. 150 cc. Temp. $60^{\circ}$

\begin{tabular}{c|c|c|c}
\hline \hline Volts & Milli-amperes & Volts & Milli-amperes \\
\cline { 2 - 3 } 0.4 & O.I & I.9 & $100^{1}$ \\
0.8 & O.I5 & 2.15 & 275 \\
I.4 & I I & 2.3 & 475.
\end{tabular}

${ }^{1}$ Deposit of metal; bubbles at anode.

$20 \mathrm{cc}_{3} \mathrm{PO}_{4}$ are necessary to redissolve the precipitate. The coating of metal is good and adherent. 
TABLE XXXIII.

Solution : $50 \mathrm{cc} \mathrm{Na} \mathrm{HPO}_{4}+\mathrm{I}_{5} \mathrm{cc}_{3} \mathrm{PO}_{4}+$ o. I g Sb as $\mathrm{SbCl}_{3}$ Vol. I 50 cc. Temp. $60^{\circ}$

\begin{tabular}{c|c||c|c}
\hline Volts & Milli-amperes & Volts & Milli-amperes \\
\hline I.3 & I I & I5 & 2.0 \\
I.8 & I.4 & $90^{1}$ \\
\end{tabular}

1 Deposit of metal; bubbles at anode.

Antimony phosphate is soluble in the amount of phosphoric acid added at $20^{\circ}$. The coating of metal is good.

TABLE XXXIV.

Solution : $50 \mathrm{cc} \mathrm{Na}_{2} \mathrm{HPO}_{4}+{ }_{5} \mathrm{cc} \mathrm{H}_{3} \mathrm{PO}_{4}+$ o.I g Pb as $\mathrm{Pb}\left(\mathrm{NO}_{3}\right)_{2}$ Vol, 150 cc. Temp. $60^{\circ}$

\begin{tabular}{l|l||l|c}
\hline Volts & Milli-amperes & Volts & Milli-amperes \\
\hline O.8 & 0.13 & 2.25 & 55 \\
I.25 & 3.5 & 2.45 & I60 \\
I.75 & I5 & 2.85 & $425^{2}$ \\
2.0 & $35^{1}$ & 3.2 & $600^{3}$
\end{tabular}

1 Bubbles at both electrodes.

2 Traces of metal.

${ }^{3}$ More metal.

Lead phosphate is soluble in the amount of phosphoric acid added at $20^{\circ}$.

TABLE XXXV.

Solution: $50 \mathrm{cc} \mathrm{Na}_{2} \mathrm{HPO}_{4}+\mathrm{I}_{5} \mathrm{cc} \mathrm{H}_{8} \mathrm{PO}_{4}+0 . \mathrm{I} \mathrm{g} \mathrm{Cd}$ as $\mathrm{CdSO}_{4}$ Vol. $150 \mathrm{cc}$. Temp. $60^{\circ}$

\begin{tabular}{l|c||c|c}
\hline \hline Volts & Milli-amperes & Volts & Milli-amperes \\
\cline { 3 - 4 } \cline { 3 - 4 } I.47 & I2 & 2.4 & 275 \\
I.85 & 20 & 2.8 & 600 \\
2.I & I40 & 2.9 & $700^{2}$
\end{tabular}

${ }^{1}$ Bubbles at both electrodes.

${ }^{2}$ Traces of metal. at $20^{\circ}$.

Cadmium phosphate is soluble in the amount of phosphoric acid added 
TABLE XXXVI.

Solution : $50 \mathrm{cc} \mathrm{Na}_{2} \mathrm{HPO}_{4}+\mathrm{I}_{5} \mathrm{cc} \mathrm{H}_{3} \mathrm{PO}_{4}+$ o. I g $\mathrm{Zn}$ as $\mathrm{ZnSO}_{4}$ Vol. I50 cc. Temp. $60^{\circ}$. Cathode copper-plated

\begin{tabular}{l|c||c|c}
\hline Volts & Milli-amperes & Volts & Milli-amperes \\
\hline 0.2 & Reversecurrent & 2.0 & $30^{1}$ \\
0.4 & 0 & 2.2 & 90 \\
0.85 & 0.3 & 2.4 & 170 \\
1.2 & 1.0 & 2.75 & $425^{2}$ \\
1.5 & I0 & 3.55 & $500^{3}$
\end{tabular}

${ }^{1}$ Bubbles at anode.

${ }^{2}$ Bubbles at cathode.

${ }^{y}$ Excellent deposit of metal.

Zinc phosphate is soluble in the amount of phosphoric acid added at $20^{\circ}$.

TABLE XXXVII.

Solution : $50 \mathrm{cc} \mathrm{Na} \mathrm{HPO}_{4}+\mathrm{I}_{5} \mathrm{cc} \mathrm{H}_{3} \mathrm{PO}_{4}+$ o. I. g Co as CoSO Vol. I 50 cc. Temp. $60^{\circ}$

\begin{tabular}{l|c|c|c}
\hline Volts & Milli-amperes & Volts & Milli-amperes \\
\hline 1.7 & I5 & 2.7 & 650 \\
1.95 & $135^{1}$ & 2.9 & 1000 \\
2.3 & 360 & 3.5 & $1750^{2}$
\end{tabular}

${ }^{1}$ Bubbles at both electrodes.

${ }^{2}$ No deposit of metal.

Cobalt phosphate is soluble in the amount of phosphoric acid added at $20^{\circ}$.

TABLE XXXVIII.

Solution : $50 \mathrm{cc} \mathrm{Na}_{2} \mathrm{HPO}_{4}+\mathrm{I}_{5} \mathrm{cc} \mathrm{H}_{9} \mathrm{PO}_{4}+$ O. I g Ni as $\mathrm{NiSO}_{4}$ Vol. $150 \mathrm{cc}$. Temp. $60^{\circ}$

\begin{tabular}{c|c||c|c}
\hline Volts & Milli-amperes & Volts & Milli-amperes \\
\hline 1.25 & 6.5 & & 2.65 \\
1.75 & 20 & 2.9 & 700 \\
1.9 & $75^{1}$ & 3.3 & 1000 \\
2.3 & 400 & 3.5 & 1300 \\
\end{tabular}

${ }^{1}$ Bubbles at both electrodes.

2 No deposit of metal.

Nickel phosphate is soluble in the amount of phosphoric acid added at $20^{\circ}$. 
TABLE XXXIX.

Solution : 50 cc. $\mathrm{Na}_{2} \mathrm{HPO}_{4}+\mathrm{I}_{5} \mathrm{cc} \mathrm{H}_{3} \mathrm{PO}_{4}+$ O. I g Sn as $\mathrm{SnCl}_{2}$ Vol. I 70 cc. Temp. $60^{\circ}$

\begin{tabular}{c|c||l|c}
\hline Volts & Milli-amperes & Volts & Milli-amperes \\
\hline I.6 & I2 & 2.5 & 525 \\
2.0 & $90^{1}$ & 2.95 & 850 \\
2.2 & 225 & 3.3 & $1000^{2}$
\end{tabular}

1 Bubbles at both electrodes.

${ }^{2}$ No deposit of metal.

Tin phosphate is insoluble at $20^{\circ}$.

Since it is not clear from any published experiments what part the sodium phosphate plays in the phosphate separations, it was thought best to make some measurements with and without sodium phosphate. These are given in Tables XL-XLVIII. It also seemed desirable to decrease the amount of free phosphoric acid.

TABLE XL.

Solution A: $6 \mathrm{cc} \mathrm{H}_{3} \mathrm{PO}_{4}+$ o. I g Ag as $\mathrm{AgNO}_{3}$

Solution B: A + 5O cc $\mathrm{Na}_{2} \mathrm{HPO}_{4}$

Vol. A r 30 cc, B I7o cc. Temp. $60^{\circ}$

\begin{tabular}{c|c|c|c}
\hline \multicolumn{2}{c|}{ A } & \multicolumn{2}{|c}{ B } \\
\hline Volts & Milli-amperes & Volts & Milli-amperes \\
\hline 0.4 & 0.05 & 0.8 & 0.15 \\
0.8 & 0.15 & $\begin{array}{l}1.3 \\
40 \\
50^{1}\end{array}$ \\
1.3 & $60^{1}$ & 1.35 &
\end{tabular}

${ }^{1}$ Deposit of metal; bubbles at anode. 
TABLE XLI.

Solution A: $6 \mathrm{cc} \mathrm{H}_{3} \mathrm{PO}_{4}+0 . \mathrm{I} \mathrm{g} \mathrm{Hg}$ as $\mathrm{HgCl}_{2}$ Solution B : A + $50 \mathrm{cc} \mathrm{Na}_{2} \mathrm{HPO}_{4}$ Vol. A I $30 \mathrm{cc}, \mathrm{B}$ I $70 \mathrm{cc}$. Temp. $60^{\circ}$

\begin{tabular}{c|c||c|c}
\hline \multicolumn{2}{c|}{ A } & \multicolumn{2}{|c}{ B } \\
\hline Volts & Milli-amperes & Volts & Milli-amperes \\
\hline 0.4 & 0.05 & 0.8 & 1.0 \\
0.84 & 0.6 & 1.2 & 35 \\
1.3 & $45^{1}$ & 1.3 & $50^{1}$ \\
\hline
\end{tabular}

${ }^{1}$ Deposit of metal; bubbles at anode.

TABLE XIII.

Solution A: $6 \mathrm{cc} \mathrm{H}_{3} \mathrm{PO}_{4}+$ o. I g Cu as $\mathrm{CuSO}_{4}$

Solution B : A + 50 cc $\mathrm{Na}_{2} \mathrm{HPO}_{4}$ Vol. A I $30 \mathrm{cc}, \mathrm{B}$ i $70 \mathrm{cc}$. Temp. $60^{\circ}$

\begin{tabular}{l|c||c|c}
\hline \multicolumn{2}{c|}{ A } & \multicolumn{2}{|c}{ B } \\
\cline { 4 - 4 } Volts & Milli-amperes & Volts & Milli-amperes \\
\hline 0.9 & 0.1 & 1.3 & 7 \\
I.35 & I 2 & 1.5 & 50 \\
I.55 & $40^{1}$ & 1.6 & $60^{1}$ \\
2.1 & $125^{2}$ & 1.8 & 100
\end{tabular}

${ }^{1}$ Good deposit of metal; bubbles at anode.

2 Bubbles at both electrodes.

TABLE XLIII.

Solution A: $6 \mathrm{cc}_{3} \mathrm{PO}_{4}$ + O. I g Bi as $\mathrm{Bi}\left(\mathrm{NO}_{8}\right)_{8}$ Solution B : A + $50 \mathrm{cc} \mathrm{Na}{ }_{2} \mathrm{HPO}_{4}$ Vol. A r $30 \mathrm{cc}, \mathrm{B}$ i $70 \mathrm{cc}$. Temp. $60^{\circ}$

\begin{tabular}{c|c||c|c}
\hline \multicolumn{2}{c|}{ A } & \multicolumn{2}{|c}{$\mathrm{B}$} \\
\hline Volts & Milli-amperes & Volts & Milli-amperes \\
\hline 0.8 & 0.4 & 1.4 & 12 \\
1.4 & 7.0 & 1.9 & $60^{1}$ \\
1.9 & $60^{i}$ & 2.4 & 300
\end{tabular}

${ }^{1}$ Good deposit of metal; bubbles at anode. 
TABLE XLIV.

Solution A: $6 \mathrm{cc}_{3} \mathrm{PO}_{4}+$ o. I g Sb as $\mathrm{SbCl}_{3}$ Solution $\mathrm{B}: \mathrm{A}+5 \mathrm{O} \mathrm{cc} \mathrm{Na}_{2} \mathrm{HPO}_{4}$

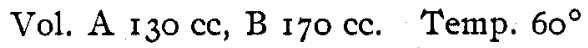

\begin{tabular}{c|c||c|c}
\hline \multicolumn{2}{c|}{$\mathrm{A}$} & \multicolumn{2}{|c}{$\mathrm{B}$} \\
\hline Volts & Milli-amperes & Volts & Milli-amperes \\
\hline I.4 & 3 & 1.4 & 4 \\
1.9 & 12 & 2.0 & $60^{1}$ \\
2.2 & $100^{1}$ & 2.2 & 100 \\
2.6 & 550 & &
\end{tabular}

${ }^{1}$ Deposit of metal ; bubbles at anode.

Metal powdery and not very adherent.

TABLE XLV.

Solution A: $6 \mathrm{cc} \mathrm{H}_{3} \mathrm{PO}_{4}+$ o. I $\mathrm{g} \mathrm{Pb}$ as $\mathrm{Pb}\left(\mathrm{NO}_{3}\right)_{2}$

Solution B: A + $50 \mathrm{oc} \mathrm{Na}_{2} \mathrm{HPO}_{4}$

Vol. A I $30 \mathrm{cc}, \mathrm{B}$ I $70 \mathrm{cc}$. Temp. $60^{\circ}$

\begin{tabular}{|c|c|c|c|}
\hline \multicolumn{2}{|c|}{$A$} & \multicolumn{2}{|c|}{$\mathrm{B}$} \\
\hline Volts & Milli-amperes & Volts & Milli-amperes \\
\hline 1.3 & I 2 & I. 3 & 8 \\
\hline 2.1 & $60^{1}$ & 2.0 & I 5 \\
\hline 2.5 & $160^{2}$ & 2.2 & $40^{3}$ \\
\hline 2.7 & 275 & 2.5 & 100 \\
\hline
\end{tabular}

1 Bubbles at electrodes.

2 Deposit of metal.

${ }^{3}$ Bubbles at anode; metal at cathode; traces of peroxide at anode. 
TABLE XLVI.

Solution A: $6 \mathrm{cc} \mathrm{H}_{3} \mathrm{PO}_{4}+$ O. I Co as $\mathrm{CoSO}_{4}$

Solution B : A + $50 \mathrm{cc} \mathrm{Na}_{2} \mathrm{HPO}_{4}$ Vol. A I 30 cc, B I 70 cc. Temp. $60^{\circ}$

\begin{tabular}{c|c||c|c}
\hline \multicolumn{2}{c|}{ A } & \multicolumn{2}{|c}{ B } \\
\hline Volts & Milli-amperes & Volts & Milli-amperes \\
\cline { 2 - 3 } & I2 & I.7 & I5 \\
1.6 & I50 & 2.1 & I $50^{1}$ \\
2.1 & 400 & 2.5 & 300 \\
2.5 & $55^{1}$ & 2.9 & $550^{2}$ \\
2.8 & $650^{3}$ & 3.5 & 650 \\
3.4 & & &
\end{tabular}

1 Bubbles at both electrodes.

2 Traces of metal.

${ }^{3}$ No deposit of metal.

TABLE XLVII.

Solution A : $6 \mathrm{cc} \mathrm{H}_{3} \mathrm{PO}_{4}$ + o. I g. $\mathrm{Ni}$ as $\mathrm{NiSO}_{4}$

Solution B : A $50 \mathrm{Occ} \mathrm{Na}_{2} \mathrm{HPO}_{4}$

Vol. A г $30 \mathrm{cc}, \mathrm{B}$ г 70 cc. Temp. $60^{\circ}$

\begin{tabular}{c|c||c|c}
\hline \multicolumn{2}{c|}{ A } & \multicolumn{2}{|c}{$\mathrm{B}$} \\
\hline Volts & Milli-amperes & Volts & Milli-amperes \\
\hline 1.7 & 20 & 2.1 & 75 \\
2.0 & 40 & 2.3 & $150^{1}$ \\
2.4 & $120^{1}$ & 2.7 & 325 \\
2.8 & 580 & 3.1 & 500 \\
3.4 & $800^{2}$ & 3.5 & 700
\end{tabular}

1 Bubbles at both electrodes.

${ }^{2}$ No deposit of metal. 
TABLE XLVIII.

Solution A: 6 cc $_{3} \mathrm{PO}_{4}+$ o. I g Sn as $\mathrm{SuCl}_{2}$ Solution B : A + 50 cc $\mathrm{Na}_{2} \mathrm{HPO}_{4}$ Vol. A I $30 \mathrm{cc}, \mathrm{B}$ I $70 \mathrm{cc}$. Temp. $60^{\circ}$

\begin{tabular}{c|c|c|c}
\hline \multicolumn{2}{c|}{$\mathrm{A}$} & \multicolumn{2}{|c}{$\mathrm{B}$} \\
\hline Volts & Milli-amperes & Volts & Milli-amperes \\
\hline I.4 & I 2 & & 2 \\
2.1 & $\mathrm{I} 50^{1}$ & 0.85 & 3 \\
2.3 & 240 & 1.4 & $60^{1}$ \\
2.7 & $45^{\circ}$ & 1.9 & 225 \\
3.15 & $600^{2}$ & 2.3 & $600^{2}$ \\
\hline
\end{tabular}

${ }^{1}$ Bubbles at both electrodes.

${ }^{2}$ No deposit of metal.

From these experiments, it is certain that the sodium phosphate is of no importance in making complex salts and changing the relative order of precipitation. Since the deposit is equally good from a phosphoric acid solution, it seems probable that the addition of sodium phosphate is an unnecessary complication. Professor Edgar F. Smith, of the University of Pennsylvania, says that they have already made determinations in his laboratory without using sodium phosphate. It is also by no means certain that the amount of free phosphoric acid now prescribed is at all necessary. The general conclusion to be drawn from my measurements is that all the phosphate separations need revision.

Since a number of separations have been made in tartrate solutions, some measturements have been made with these solutions. In each case the tartrate solution contained $7.5 \mathrm{~g}$ crystallized tartaric acid and $35 \mathrm{cc}$ aqueous ammonia sp. gr. 0.92 . The results are given in Tables XLIX-LIX. 
TABLE XLIX.

Solution : amm. tartrate

Vol, r 70 cc. Temp. $60^{\circ}$

\begin{tabular}{c|c||c|c}
\hline \hline Volts & Milli-amperes & Volts & Milli-amperes \\
\cline { 2 - 3 } 0.2 & 1.0 & 1.85 & 75 \\
0.4 & 4.5 & 1.9 & $85^{1}$ \\
0.8 & 10 & 2.0 & $125^{2}$ \\
1.3 & 18 & 2.35 & 450 \\
I.7 & 45 & &
\end{tabular}

1 Bubbles at cathode.

2 Bubbles at anode.

TABLF L.

Solution : amm. tartrate + o. I g Ag as $\mathrm{AgNO}_{3}$ Vol. 170 cc. Temp. $60^{\circ}$

\begin{tabular}{c|c|c|c}
\hline Volts & Milli-amperes & Volts & Milli-amperes \\
\cline { 2 - 3 } 0.4 & 0.8 & 1.0 & $75^{1}$ \\
0.8 & 60 & 1.5 & 75
\end{tabular}

${ }^{1}$ Deposit of metal; bubbles at anode.

TABLE LI.

Solution : amm. tartrate + o. $\mathrm{g} \mathrm{Hg}$ as $\mathrm{HgCl}_{2}$ Vol. I 70 cc. Temp. $60^{\circ}$

\begin{tabular}{c|c||c|c}
\hline \hline Volts & Milli-amperes & Volts & Milli-amperes \\
\cline { 1 - 2 } 0.9 & 35 & I.6 & 65 \\
I.2 & $65^{1}$ & 2.1 & I 50
\end{tabular}

${ }^{1}$ Deposit of metal; bubbles at anode. 
TABLE LII.

Solution : amm. tartrate + o. I g Pb as $\mathrm{Pb}\left(\mathrm{NO}_{3}\right)_{2}$

Vol. 170 cc. Temp. $60^{\circ}$

\begin{tabular}{c|c|c|c}
\hline Volts & Milli-amperes & Volts & Milli-amperes \\
\hline 0.8 & $\mathrm{I} 2$ & $\begin{array}{l}1.4 \\
1.9\end{array}$ & $50^{2}$ \\
1.3 & $40^{\prime}$ & $80^{8}$
\end{tabular}

1 Deposit of metal.

${ }^{2}$ No bubbles at anode.

2 Bubbles at both electrodes.

Some peroxide deposits at anode. Deposit at cathode good and adherent.

TABLE LIII.

Solution : amm. tartrate + o. I $\mathrm{g} \mathrm{Bi}$ as $\mathrm{Bi}\left(\mathrm{NO}_{3}\right)_{3}$

Vol. 170 cc. Temp. $60^{\circ}$

\begin{tabular}{l|c|c|c}
\hline \hline Volts & Milli-amperes & Volts & Milli-amperes \\
\hline 0.8 & I I & I.7 & 40 \\
I.25 & 35 & 2.0 & 65 \\
I.35 & $40^{1}$ & 2.3 & $225^{2}$
\end{tabular}

${ }^{1}$ Deposit of metal ; no bubbles at either electrode.

2 Bubbles at both electrodes.

Very good deposit of metal at cathode.

TABLE LIV.

Solution : amm. tartrate + o. I $\mathrm{g} \mathrm{Cu}$ as $\mathrm{CuSO}_{4}$ Vol. I7o cc. Temp. $60^{\circ}$

\begin{tabular}{l|c|c|c}
\hline Volts & Milli-amperes & Volts & Milli-amperes \\
\hline I.25 & 80 & 1.75 & 160 \\
1.4 & 90 & 2.0 & $250^{2}$ \\
1.65 & $160^{1}$ & 2.3 & 550
\end{tabular}

1 Traces of copper.

2 Bubbles at both electrodes.

Copper deposit not good. 


\section{TABLE LV.}

Solution: amm. tartrate + O.I $\mathrm{g}$ Co as $\mathrm{CoSO}_{4}$ Vol. $170 \mathrm{cc}$. Temp. $60^{\circ}$

\begin{tabular}{l|c||c|c}
\hline Volts & Milli-amperes & Volts & Milli-amperes \\
\cline { 2 - 3 } 0.9 & 6 & 1.8 & 60 \\
I.35 & 15 & 2.0 & $150^{1}$
\end{tabular}

${ }^{1}$ Bubbles at both electrodes ; metal at cathode; deposit good.

TABLE LVI.

Solution : amm. tartrate + O. $\mathrm{g} \mathrm{Cd}$ as $\mathrm{CdSO}_{4}$ Vol. $170 \mathrm{cc}$. Temp. $60^{\circ}$

\begin{tabular}{c|c||c|c}
\hline Volts & Milli-amperes & Volts & Milli-amperes \\
\hline I.7 & 22 & 2.2 & I 25 \\
2.1 & $80^{1}$ & 2.3 & I 25
\end{tabular}
kept low.

${ }^{1}$ Deposit of metal; bubbles at anode. Deposit is good if voltage is TABLE LVII.

Solution : amm. tartrate $+0 . \mathrm{I} \mathrm{g} \mathrm{Zn}$ as $\mathrm{ZnSO}_{4}$ Vol. I70 cc. Temp. $60^{\circ}$. Cathode copper-plated

\begin{tabular}{c|c|c|c}
\hline \hline Volts & Milli-amperes & Volts & Milli-amperes \\
\hline 0.2 & Reverse current & I.7 & I5 \\
0.4 & 0.1 & 2.2 & 22 \\
0.8 & I.8 & 2.3 & $70^{1}$ \\
1.3 & IO & 2.5 & 160
\end{tabular}

${ }^{1}$ Deposit of metal; bubbles at anode.

TABLE LVIII.

Solution : amm. tartrate + O. I g Ni as $\mathrm{NiSO}_{4}$ Vol. I70 cc. Temp. $60^{\circ}$

\begin{tabular}{c|c||c|c}
\hline Volts & Milli-amperes & Volts & Milli-amperes \\
\hline 0.85 & Io & $\begin{array}{l}\text { I.85 } \\
2.3\end{array}$ & I $25^{1}$ \\
I.35 & I5 & $600^{2}$
\end{tabular}

1 Bubbles at cathode; none at anode.

${ }^{2}$ Deposit of metal and bubbles at each electrode. 
TABLE LIX.

Solution : amm, tartrate + o. I g $\mathrm{Sn}$ as $\mathrm{SnCl}_{2}$ Vol. I 70 cc. Temp. $60^{\circ}$

\begin{tabular}{c|c|c|c}
\hline Volts & Milli-amperes & Volts & Milli-amperes \\
\cline { 2 - 4 } & 25 & 2.5 & 375 \\
I.65 & $75^{1}$ & 3.0 & 650 \\
2.9 & $\mathrm{I} 30$ & 3.5 & I $200^{2}$
\end{tabular}

${ }^{1}$ Bubbles at both electrodes.

${ }^{2}$ No deposit of metal.

From Neumann's measurements of the electromotive force, ${ }^{\mathrm{x}}$ we know the voltaic series in chloride, nitrate, and sulphate solutions. His experiments on hydrogen refer to a normal acid, and it seemed desirable to have the data on file showing that zinc precipitates before hydrogen in a neutral solution. Experiments were therefore made with zinc silphate, magnesium sulphate, and sodium sulphate, the first giving metal, the second hydrogen and a sparingly soluble base, and the third hydrogen and a soluble base. All these series were made with a coppered cathode so that they might be comparable.

TABLE LX.

Solution : I mol $\mathrm{ZnSO}_{4}$ per liter Vol. $200 \mathrm{cc}$. Temp. $20^{\circ}$. Cathode copper-plated

\begin{tabular}{c|c|c|c}
\hline Volts & Milli-amperes & Volts & Milli-amperes \\
\hline 0.8 & 0.I & 2.3 & I2 \\
I.4 & I.O & 2.5 & I 5 \\
I.7 & 2.0 & 2.75 & I $25^{1}$
\end{tabular}

${ }^{1}$ Metal at cathode; bubbles at anode.

1 Zeit. phys. Chem. 14, 229 (1897). 
TABLE L XI.

Solution : I mol $\mathrm{MgSO}_{4}$ per liter

Vol. $200 \mathrm{cc}$. Temp. $20^{\circ}$. Cathode copper-plated

\begin{tabular}{c|c||c|c}
\hline \hline Volts & Milli-amperes & Volts & Milli-amperes \\
\hline I.3 & 0.4 & 2.75 & $40^{1}$ \\
I.7 & IO & 3.1 & $160^{2}$ \\
2.5 & I2 & 3.5 & 410
\end{tabular}

I Bubbles at anode.

2 Bubbles at cathode.

TABLE LXII.

Solution : r mol $\mathrm{Na}_{2} \mathrm{SO}_{4}$ per liter

Vol. 200 cc. Temp. $20^{\circ}$. Cathode copper-plated

\begin{tabular}{l|c||c|c}
\hline \hline Volts & Milli-amperes & Volts & Milli-amperes \\
\cline { 2 - 4 } I.7 & 2.0 & 3.0 & $100^{1}$ \\
2.5 & I0 & 3.35 & $350^{2}$ \\
2.75 & I5 & 3.5 & 525
\end{tabular}

${ }^{2}$ Bubbles at anode.

${ }^{2}$ Bubbles at cathode.

A convenient method for determining nickel is the one using a solution of ammonium sulphate and ammonia. Since cadmium can be separated from nickel in a solution containing ammonium sulphate and sulphuric acid, it seemed necessary to make some measurements with cadmium and nickel in ammonium sulphate solutions to which ammonia or sulphuric acid had been added. The results obtained are given in Tables I.VIII-LXXI. 
TABLE LXIII.

Solution : $\operatorname{rog}\left(\mathrm{NH}_{4}\right)_{2} \mathrm{SO}_{4}+30 \mathrm{Oc} \mathrm{NH} \mathrm{NH}_{4}(0.92)+0.2 \mathrm{~g} \mathrm{Ni}$ as $\mathrm{NiSO}_{4}$ Vol. 2,00 cc. Temp. $20^{\circ}$

\begin{tabular}{l|c||c|c}
\hline \hline Volts & Milli-amperes & Volts & Milli-amperes \\
\cline { 2 - 3 } & 0.6 & & $600^{2}$ \\
O.76 & 3.0 & 2.35 & 1000 \\
I.I5 & 3.8 & 2.75 & $1250^{3}$ \\
I.5 & $75^{1}$ & 2.95 & 1570 \\
I.85 & 365 & 3.25 & 2080 \\
2.15 & 3.5 &
\end{tabular}

1 Bubbles at anode.

${ }^{2}$ Bubbles at both electrodes.

${ }^{3}$ Deposit of metal.

\section{TABLE LXIV.}

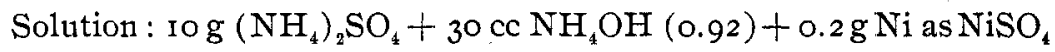
Vol. $200 \mathrm{cc}$. Temp. $60^{\circ}$

\begin{tabular}{l|c|c|c}
\hline Volts & Milli-amperes & Volts & Milli-amperes \\
\cline { 2 - 3 } & 2.4 & 2.0 & $325^{1}$ \\
1.15 & 10 & 2.1 & 550 \\
I.6 & I8 & 2.4 & 900
\end{tabular}

${ }^{1}$ Deposit of metal; bubbles at anode.

TABLE LXV.

Solution: $\log \left(\mathrm{NH}_{4}\right)_{2} \mathrm{SO}_{4}+30 \mathrm{cc} \mathrm{NH} \mathrm{NH}_{4} \mathrm{OH}(0.92)+0.2 \mathrm{~g} \mathrm{Cd}$ as $\mathrm{CdSO}_{4}$ Vol. 200 cc. Temp. $20^{\circ}$

\begin{tabular}{c|c||c|c}
\hline \hline Volts & Milli-amperes & Volts & Milli-amperes \\
\hline I.2 & 2.5 & 2.4 & $85^{2}$ \\
I.7 & 3.7 & 2.85 & I85 \\
2.I & $25^{\mathrm{i}}$ & 3.25 & 650
\end{tabular}

${ }^{1}$ Bubbles at anode.

${ }^{2}$ Heavy deposit of metal. 


\section{TABLE LXVI.}

Solution: $\log \left(\mathrm{NH}_{4}\right)_{2} \mathrm{SO}_{4}+30 \mathrm{Oc} \mathrm{NH}_{4} \mathrm{OH}(0.92)+0.2 \mathrm{~g} \mathrm{Cd}$ as $\mathrm{CdSO}_{4}$ Vol, 200 cc. Temp. $60^{\circ}$

\begin{tabular}{l|c||c|c}
\hline Volts & Milli-amperes & Volts & Milli-amperes \\
\hline 0.45 & 0.2 & I .8 & I 5 \\
0.9 & I.5 & 2.3 & I $75^{1}$ \\
I.45 & I 2 & 2.64 & 4 IO
\end{tabular}

${ }^{1}$ Heavy deposit of metal; bubbles at anode.

TABLE LXVII.

Solution: $\log \left(\mathrm{NH}_{4}\right)_{2} \mathrm{SO}_{4}+4 \mathrm{cc} \mathrm{H}_{2} \mathrm{SO}_{4}(\mathrm{r} .09)+0.2 \mathrm{~g} \mathrm{Ni}$ as $\mathrm{NiSO}_{4}$ Vol. $200 \mathrm{cc}$. Temp. $20^{\circ}$

\begin{tabular}{l|c|l|c}
\hline Volts & Milli-amperes & Volts & Milli-amperes \\
\hline 0.76 & 0.15 & 2.7 & $420^{2}$ \\
I.0 & 1.5 & 3.05 & 680 \\
I.4 & 2.4 & 3.4 & 1050 \\
2.0 & $85^{3}$ & 3.65 & 1300 \\
2.35 & 160 & 4.4 & 2750
\end{tabular}

${ }^{1}$ Bubbles at both electrodes.

${ }^{2}$ Very slight deposit of metal.

TABLE LXVIII.

Solution: $\operatorname{rog}\left(\mathrm{NH}_{4}\right)_{2} \mathrm{SO}_{4}+4 \mathrm{cc} \mathrm{H}_{2} \mathrm{SO}_{4}(\mathrm{r} .09)+0.2 \mathrm{~g} \mathrm{Ni}$ as $\mathrm{NiSO}_{4}$ Vol. $200 \mathrm{cc}$. Temp. $60^{\circ}$

\begin{tabular}{l|c|c|c}
\hline \hline Volts & Milli-amperes & Volts & Milli-amperes \\
\hline I.2 & $\mathrm{I} .9$ & 2.6 & 800 \\
1.95 & $75^{1}$ & 2.8 & $1250^{3}$ \\
2.2 & $265^{2}$ & 3.1 & 2000
\end{tabular}

${ }^{1}$ Slight bubbles at anode.

${ }^{2}$ Bubbles at both electrodes.

3 Traces of metal. 
TABLE LXIX.

Solution: $\log \left(\mathrm{NH}_{4}\right)_{2} \mathrm{SO}_{4}+{ }_{4} \mathrm{cc} \mathrm{H}_{2} \mathrm{SO}_{4}$ (I.09) + $0.2 \mathrm{~g} \mathrm{Cd}$ as $\mathrm{CdSO}_{4}$ Vol. $200 \mathrm{cc}$. Temp. $20^{\circ}$

\begin{tabular}{c|c|c|c}
\hline Volts & Milli-amperes & Volts & Milli-amperes \\
\hline I.I2 & 2.9 & 2.4 & $75^{1}$ \\
I.65 & 3.9 & 2.8 & $275^{2}$ \\
2.I5 & I8 & 3.5 & 800
\end{tabular}

1 Traces of bubbles at anode.

2 Deposit of metal.

TABLE LXX.

Solution : $10 \mathrm{~g}\left(\mathrm{NH}_{4}\right)_{2} \mathrm{SO}_{4}+4 \mathrm{Cc} \mathrm{H}_{2} \mathrm{SO}_{4}(\mathrm{I} .09)+0.2 \mathrm{~g} \mathrm{Cd}$ as $\mathrm{CdSO}_{4}$ Vol. $200 \mathrm{cc}$. Temp. $60^{\circ}$

\begin{tabular}{c|c|c|c}
\hline \hline Volts & Milli-amperes & Volts & Milli-amperes \\
\hline 0.8 & 0.5 & 2.0 & 35 \\
1.26 & 5 & 2.32 & $185^{1}$ \\
1.7 & $I 2$ & 2.75 & $525^{2}$
\end{tabular}

${ }^{1}$ Slight deposit of metal; bubbles at anode.

${ }^{2}$ Good deposit of metal.

TABLE LXXI.

Solution: $\log \left(\mathrm{NH}_{4}\right)_{2} \mathrm{SO}_{4}+8 \mathrm{cc} \mathrm{H}_{2} \mathrm{SO}_{4}(\mathrm{I} .09)+0.2 \mathrm{~g} \mathrm{Ni}$ as $\mathrm{NiSO}_{4}$ Vol. $200 \mathrm{cc}$. Temp. $20^{\circ}$

\begin{tabular}{c|c|c|c}
\hline Volts & Milli-amperes & Volts & Milli-amperes \\
\hline 0.76 & O.I & 2.5 & 650 \\
I.15 & I.I & 2.85 & IO50 \\
1.65 & 3.5 & $3 . \mathrm{I}$ & I 250 \\
2.2 & $215^{1}$ & 3.7 & $2150^{2}$
\end{tabular}

${ }^{1}$ Bubbles at both electrodes.

a Slight deposit of metal.

From these experiments it seems improbable that a satisfactory separation of cadmium from nickel can be obtained when 
only $4 \mathrm{cc} \mathrm{H}_{2} \mathrm{SO}_{4}$ are added. The addition of more acid raises the voltage at which nickel precipitates and has little effect on cadmium, so that a separation is then possible. One curious phenomenon comes out even more clearly here than in the previous experiments, the effect of cadmium sulphate on the evolution of hydrogen. In Table LXVII there is visible evolution of hydrogen under a potential difference of 2.0 volts. In Table LXIX there is no perceptible evolution of hydrogen at 2.8 volts. The first solution contains $0.2 \mathrm{~g} \mathrm{Ni}$ as $\mathrm{NiSO}_{4}$, the second $0.2 \mathrm{~g}$ $\mathrm{Cd}$ as $\mathrm{CdSO}_{4}$; otherwise there is no difference between them.

From the tables given in this paper and from some that have not been published, I have compiled tables showing the voltaic series for each solution and the approximate voltage at which each metal appears under the conditions of the experiments. The values for zinc are not strictly comparable with those for the other metals because they were made with a copper cathode, and the potential difference $\mathrm{Cu}-\mathrm{Pt}$ should be added. In cases where no precipitation of metal was obtained, the value is said to be higher than the highest voltage actually measured.

For the sake of completeness, the voltaic series for normal solutions of the metals as ions is given in Table LXXII. This is taken from Neumann's experiments ${ }^{x}$ and, barring insoluble salts, holds for chlorides, nitrates and sulphates. It must be remembered that electromotive force measurements and electrolysis measurements will give the same order only in case the reaction at the electrode is strictly reversible, which is possibly not true for nickel.

TABLE LXXII.

Voltaic series in normal solutions

\begin{tabular}{l|l}
\hline \hline $\mathrm{Ag}$ & $\mathrm{Sn}$ \\
$\mathrm{Hg}$ & $\mathrm{Co}$ \\
$\mathrm{Cu}$ & $\mathrm{Ni}$ \\
$\mathrm{Sb}$ & $\mathrm{Fe}$ \\
$\mathrm{Bi}$ & $\mathrm{Cd}$ \\
$\mathrm{H}\left(\mathrm{H}_{2} \mathrm{SO}_{4}\right)$ & $\mathrm{Zn}$ \\
$\mathrm{Pb}$ & $\mathrm{H}(\mathrm{NaOH})$ \\
\hline
\end{tabular}

${ }^{1}$ Zeit. phys. Chem, 14, 223 (1894). 
Electrochemical Analysis and the Voltaic Series $\quad 46 \mathrm{r}$

TABLE LXXIII.

Voltaic series in $\mathrm{N} / 4 \mathrm{KCN}+0.2 \mathrm{~g}$ metal per $200 \mathrm{cc}$

\begin{tabular}{ll|ll}
\hline \multicolumn{2}{c|}{ Temp. $20^{\circ}$} & \multicolumn{2}{c}{ Temp. 60 } \\
\hline $\mathrm{Ag}$ & 2.04 & $\mathrm{Ag}$ & $\mathrm{I} .8$ \\
$\mathrm{Hg}$ & 2.2 & $\mathrm{Hg}$ & 2.0 \\
$\mathrm{Bi}$ & 2.5 & $\mathrm{H}$ & 2.5 \\
$\mathrm{H}$ & 2.74 & $\mathrm{Cu}$ & 2.6 \\
$\mathrm{Cd}$ & 2.8 & $\mathrm{Cd}$ & 2.8 \\
$\mathrm{Zn}$ & $>4.5$ & $\mathrm{Zn}$ & $>4 . \mathrm{I}$ \\
$\mathrm{Ni}$ & $>4.2$ & $\mathrm{Ni}$ & $>3.5$ \\
$\mathrm{Cu}$ & $>4.4$ & &
\end{tabular}

TABLE LXXIV.

Voltaic series in $2 \mathrm{~N} / \mathrm{1} \mathrm{KCN}+0.2 \mathrm{~g}$ metal per $200 \mathrm{cc}$

\begin{tabular}{ll|ll}
\hline \multicolumn{2}{c|}{ Temp. $20^{\circ}$} & \multicolumn{2}{c}{ Temp. $60^{\circ}$} \\
\hline $\mathrm{Hg}$ & 1.98 & $\mathrm{Ag}$ & \multicolumn{1}{c}{1.67} \\
$\mathrm{H}$ & 2.3 & $\mathrm{Hg}$ & 1.87 \\
$\mathrm{Cd}$ & 2.5 & $\mathrm{Cd}$ & 2.3 \\
$\mathrm{Ag}$ & 2.9 & $\mathrm{H}$ & $2.65^{1}$ \\
$\mathrm{Cu}$ & $>3.3$ & $\mathrm{Cu}$ & $>3.3$ \\
$\mathrm{Zn}$ & $>3.3$ & $\mathrm{Zn}$ & $>3.3$ \\
$\mathrm{Ni}$ & $>3.5$ & $\mathrm{Ni}$ & $>3.5$
\end{tabular}

1 This determination was made with an old solution and is probably wrong. As the value is of no interest, it was not redetermined.

TABLE, LXXV.

Voltaic series in oxalate solution + o. I g metal per $175 \mathrm{cc}$

\begin{tabular}{|c|c|c|c|}
\hline \multicolumn{2}{|c|}{ Temp. $60^{\circ}$} & \multicolumn{2}{|c|}{ Temp. $60^{\circ}$} \\
\hline $\mathrm{Hg}$ & 0.65 & $\mathrm{Cd}$ & I .8 \\
\hline $\mathrm{Cu}$ & 1.0 & $\mathrm{Ni}$ & $\mathrm{I} .9$ \\
\hline $\mathrm{Bi}$ & I. I 5 & Co & I. 9 \\
\hline $\mathrm{Sb}$ & I. 2 & $\mathrm{Fe}$ & I.9 \\
\hline $\mathrm{H}$ & I. 45 & Sn & 2.I \\
\hline $\mathrm{Pb}$ & I. 55 & $\mathrm{Zn}_{\mathrm{n}}$ & 2,6 (copper) \\
\hline
\end{tabular}




\section{TABLE LXXVI.}

Voltaic series in acid sodium phosphate solutions $50 \mathrm{cc} \mathrm{Na}_{2} \mathrm{HPO}_{4}+{ }_{5} \mathrm{cc}_{3} \mathrm{PO}_{4}+$ O. I g metal per $1.50 \mathrm{cc}$

\begin{tabular}{|c|c|c|c|}
\hline \multicolumn{2}{|c|}{ Temp. $60^{\circ}$} & \multicolumn{2}{|c|}{ Temp. $60^{\circ}$} \\
\hline $\mathrm{Ag}$ & 1.2 & $\mathrm{~Pb}$ & 2.85 \\
\hline $\mathrm{Hg}$ & $\mathrm{I} \cdot 3$ & $\mathrm{Cd}$ & 2.9 \\
\hline $\mathrm{Cu}$ & I. 75 & $\mathrm{Zn}$ & 3. I 5 (copper) \\
\hline $\mathrm{Bi}$ & I. 9 & Co & $>3 \cdot 5$ \\
\hline $\mathrm{H}$ & 1.9 & $\mathrm{Ni}$ & $>3.5$ \\
\hline $\mathrm{Sb}$ & 2.0 & Sn & $>3.3$ \\
\hline
\end{tabular}

TABLE LXXVII.

Voltaic series in phosphate solutions $6 \mathrm{cc}_{8} \mathrm{PO}_{4}+$ O. I g metal per $\mathrm{I}_{3} \mathrm{O} \mathrm{cc}$

\begin{tabular}{|c|c|c|c|}
\hline \multicolumn{2}{|c|}{ Temp. $60^{\circ}$} & \multicolumn{2}{|c|}{ Temp. $60^{\circ}$} \\
\hline $\mathrm{Ag}$ & I. 3 & $\mathrm{H}$ & 2.25 \\
\hline $\mathrm{Hg}$ & I. 3 & $\mathrm{Cd}$ & 2.4 \\
\hline $\mathrm{Cu}$ & I. 6 & $\mathrm{Zn}_{\mathrm{n}}$ & 2.7 (copper) \\
\hline $\mathrm{Bi}$ & I.9 & $\mathrm{Zn}$ & $3 \cdot 4$ (platinum) \\
\hline $\mathrm{Sb}$ & 2.1 & $\mathrm{Co}$ & 2.9 \\
\hline $\mathrm{Pb}$ & 2.2 & $\mathrm{Ni}$ & $>3.5$ \\
\hline & & $\mathrm{Sn}$ & $>3.5$ \\
\hline
\end{tabular}

TABLE XXVIII.

Voltaic series in ammoniacal tartrate $+0.1 \mathrm{~g}$ metal per $\mathrm{r} 7 \mathrm{o} \mathrm{cc}$

\begin{tabular}{ll|ll}
\hline \multicolumn{2}{c|}{ Temp. $60^{\circ}$} & \multicolumn{2}{|c}{ Temp. $60^{\circ}$} \\
\cline { 2 - 4 } $\mathrm{Ag}$ & 1.0 & \multicolumn{2}{|c}{} \\
$\mathrm{Hg}$ & 1.2 & $\mathrm{Co}$ & 2.0 \\
$\mathrm{~Pb}$ & 1.3 & $\mathrm{Cd}$ & 2.1 \\
$\mathrm{Bi}$ & 1.30 & $\mathrm{Zn}$ & 2.3 (copper) \\
$\mathrm{Cu}$ & 1.65 & $\mathrm{Ni}$ & 2.3 \\
$\mathrm{H}$ & 1.9 & $\mathrm{Sn}$ & $>4.8$
\end{tabular}


TABLE LXXIX.

Voltaic series in alkaline tartrate solution

$5 \mathrm{~g}$ cryst. sodium potassium tartrate $+25 \mathrm{cc} \mathrm{NaOH}$ (sp. gr. I. 28 at $20^{\circ}$ ) + O. I $\mathrm{g}$ metal per $\mathrm{I} 5 \mathrm{O} \mathrm{cc}$

\begin{tabular}{ll|ll}
\multicolumn{2}{c|}{ Temp. $20^{\circ}$} & \multicolumn{2}{|c}{ Temp. $20^{\circ}$} \\
\hline $\mathrm{Zn}$ & 1.7 (platinum) & $\mathrm{Bi}$ & 2.0 \\
$\mathrm{Cu}$ & $\mathrm{I} .75$ & $\mathrm{Sb}$ & 2.0 \\
$\mathrm{Co}$ & 1.75 & $\mathrm{Sn}$ & $>3.0$ \\
$\mathrm{~Pb}$ & 2.0 & $\mathrm{Ni}$ & $>2.8$ \\
$\mathrm{H}$ & 2.0 & &
\end{tabular}

These tables point to the possibility of several new separations, but we will only consider two at the present moment. From Table LXXIV it appears that the decomposition voltage for silver is nearly a volt higher than that for mercury in a double normal potassium cyanide solution at $20^{\circ}$. A series of runs showed, however, that silver was always precipitated with the mercury. This is not especially surprising since mercury precipitates as a liquid and silver and mercury are known to form compounds. When the decomposition voltages of two metals are practically the same, no separation is possible. The converse is not necessarily true that a separation can be made when the decomposition voltages are very different. This is true only when neither of the metals affects the decomposition voltage of the other, a condition which does not hold experimentally in this case.

From Tables LXXIX it appears that nickel does not precipitate from an alkaline tartrate solution while cobalt does. This points to the possibility of precipitating cobalt at the cathode free from nickel and is the method actually proposed by Vortmann. ${ }^{x}$ Some preliminary experiments indicate that this separation is more satisfactory than has hitherto been supposed.

The main object of this investigation was to obtain the data necessary for a classification of the methods of analysis now in use. In Tables LXXX-LXXXV are given most of the important separations for silver, mercury, copper, bismuth, lead,

${ }^{1}$ Monatshefte für Chemie, 14,536 ( 1893 ). 


\begin{tabular}{|c|c|c|c|}
\hline \multicolumn{4}{|c|}{ Silver or mercury from } \\
\hline $\mathrm{Cu}$ & $\begin{array}{l}\text { Nitric acid } \\
\text { Cyanide }\end{array}$ & $\mathrm{V}$ & $\mathrm{V}$ \\
\hline & $\begin{array}{l}\text { Cyanide } \\
\text { Nitric acid }\end{array}$ & $\mathrm{V}$ & $\mathrm{V}$ \\
\hline $\mathrm{Pb}$ & Excess nitric acid & $c$ & $\mathrm{c}$ \\
\hline Sn & $\begin{array}{l}\text { Sulphide } \\
\text { (AgSinsoluble) }\end{array}$ & & \\
\hline $\mathrm{Fe}$ & Nitric acid & C & C \\
\hline & Cyanide & $\mathrm{c}$ & c \\
\hline $\mathrm{Ni}$ & Acid & C & C \\
\hline & Cyanide & C & C \\
\hline $\mathrm{Cd}$ & Nitric acid & C & c \\
\hline & Cyanide & V? & C \\
\hline $\mathrm{Zn}$ & Cyanide & $\mathrm{c}$ & C \\
\hline
\end{tabular}

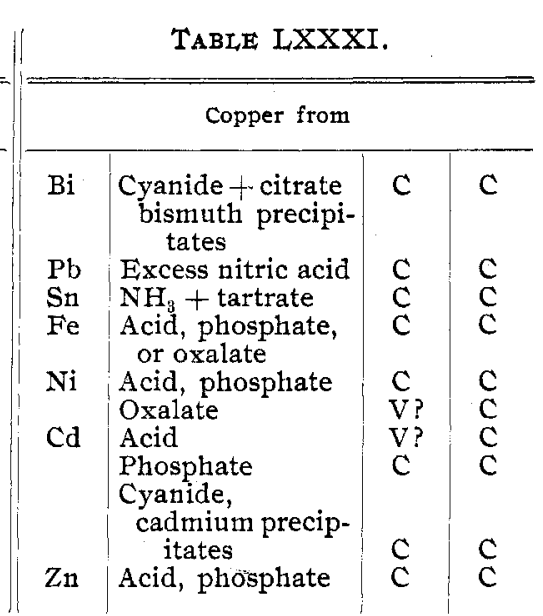

TABLE LXXXII.

\begin{tabular}{l|l|l|l|}
\hline \multicolumn{3}{|c|}{ Bismuth from } \\
\hline $\mathrm{Pb}$ & None & & \\
$\mathrm{Sn}$ & $\mathrm{NH}_{3}+$ tartrate & $\mathrm{C}$ & $\mathrm{C}$ \\
$\mathrm{Fe}$ & Acid sulphate & $\mathrm{C}$ & $\mathrm{C}$ \\
$\mathrm{Ni}$ & Acid sulphate & $\mathrm{C}$ & $\mathrm{C}$ \\
$\mathrm{Cd}$ & Acid & $\mathrm{C}$ & $\mathrm{C}$ \\
$\mathrm{Zn}$ & Acid & $\mathrm{C}$ & $\mathrm{C}$ \\
& & & \\
& & &
\end{tabular}

TABLE LXXXIII.

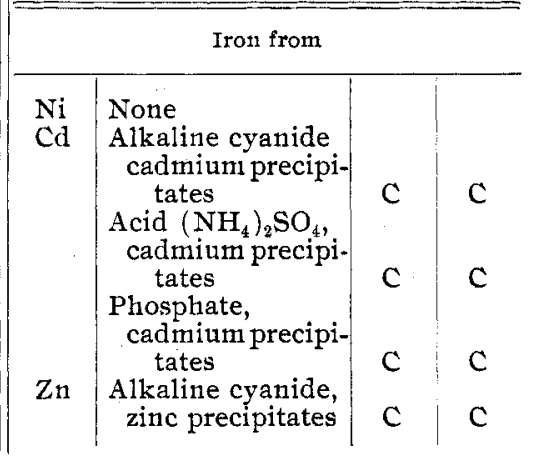

TABLE LXXXIV.

TABLE LXXXV.

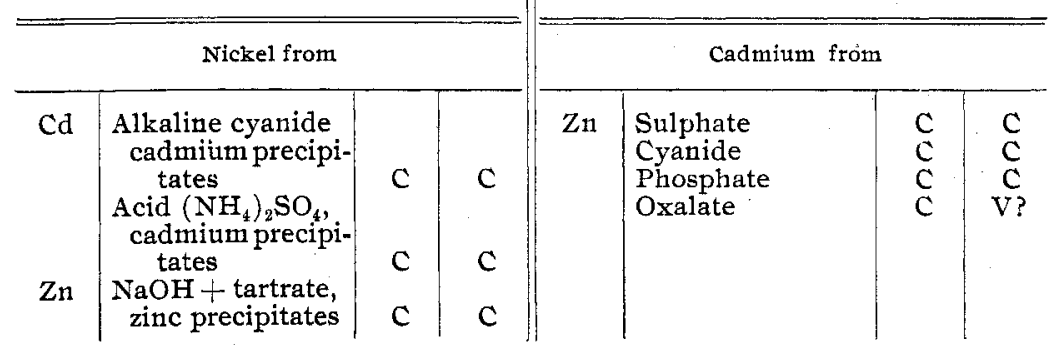

tin, nickel, iron, cadmium, and zinc. The first column gives the metal and the second the solution. In the third column $\mathrm{C}$ means that a constant current separation is used and $\mathrm{V}$ a voltage 
separation. In the fourth column the same letters refer to the method of separation as predicted from measurements of decomposition voltage. The third column thus refers to practice, and the fourth to theory. Obviously the two should agree.

As was to have been expected, practically all the determinations are constant current separations, and the few which are not are of minor importance. There are only a few cases in which there is a discrepancy between the analytical method and the results of decomposition measurements. The separation of cadmium from zinc in oxalate solutions becomes intelligible in view of the experimental fact already referred to, that cadmium precipitates before hydrogen, though at a higher voltage. This may also account for the separation of silver from cadmium in cyanide solution; but I think not. It seems more probable that the behavior of silver and cadmium in cyanide solutions, of copper and nickel in oxalate solution, and of copper and cadmium in acid solution should be referred to the formation of an alloy. It is known that the decomposition voltage is changed under these circumstances. ${ }^{x}$ On the other hand, it is also possible that it is merely a result of insufficient stirring just as $\mathrm{Smith}^{2}$ found that copper carries down iron when precipitated on a platinum dish, but does not when precipitated on a vertical cathode.

Some experiments were made on the rate of deposition when the solution was stirred vigorously. I succeeded in getting copper completely precipitated inside of twenty minutes, when the paper of Gooch and Medway ${ }^{3}$ appeared, in which a more efficient stirring had given still better results in point of time.

In this paper are given the data necessary for a systematic classification of most of the analytical methods with the exception of the sulphide solutions. The work was undertaken at the suggestion of Professor Bancroft, and has been carried under his direction.

Cornell University.

\footnotetext{
${ }^{1}$ Coehn. Zeit. phys. Chem. 38, 609 (Igor).

2 Jour. Am. Chem. Soc. 24, I073 (1902).

${ }^{3}$ Am. Jour. Sci. (4) I5, 320 (1903).
} 